\title{
Detecting the Candidate Gender Determinants by Bioinformatic Prediction of miRNAs and Their Targets from Transcriptome Sequences of the Male and Female Flowers in Salix suchowensis
}

\author{
Suyun Wei, ${ }^{1,2}$ Ning Ye, ${ }^{2}$ and Tongming Yin ${ }^{1}$ \\ ${ }^{1}$ Co-Innovation Center for Sustainable Forestry in Southern China, Nanjing Forestry University, Nanjing, China \\ ${ }^{2}$ College of Information Science and Technology, Nanjing Forestry University, Nanjing, China \\ Correspondence should be addressed to Tongming Yin; tmyin@njfu.edu.cn
}

Received 14 November 2016; Revised 4 May 2017; Accepted 8 May 2017; Published 30 May 2017

Academic Editor: Cheol Yong Choi

Copyright (c) 2017 Suyun Wei et al. This is an open access article distributed under the Creative Commons Attribution License, which permits unrestricted use, distribution, and reproduction in any medium, provided the original work is properly cited.

MicroRNAs (miRNAs) belong to a class of small, noncoding, and endogenous single-stranded RNAs that negatively regulate gene expression at the posttranscriptional level. Potential miRNAs can be identified based on sequence homology since miRNAs are highly conserved in plants. In this study, we aligned the expressed sequence tags derived from flower buds of male and female $S$. suchowensis to miRNAs in the miRBase, which enable us to identify 34 potential miRNAs from flower buds of the alternate sexes. Among them, 11 were from the female and 23 were from the male. Analyzing sequence complementarity led to identification of 124 and 55 miRNA targets in the male and female flower buds, respectively. By mapping the target genes of the predicted miRNAs to the sequence assemblies of S. suchowensis, a miR156 mediated gene was detected at the gender locus of willow, which was a transcription factor involved in flower development. It is noteworthy that this target is not expressed in male flower, while it is expressed fairly highly in female flower based on the transcriptome data derived from the alternate sexes of willows. This study provides new bioinformatic clue for further exploring the genetic mechanism underlying gender determination in willows.

\section{Introduction}

The miRNAs, approximately in length of 22-nucleotides, have been widely detected in diverse organisms. They are noncoding, endogenous, and single-stranded small RNAs, which negatively regulate gene expression in plants and animals at the posttranscriptional level $[1,2]$ by mediating target mRNA cleavage $[3,4]$ or translational repression $[5,6]$. Increasing evidence suggests that plant miRNAs have several crucial roles in gene regulatory networks. They are believed to be involved in multiple plant developmental and metabolic pathways affecting developmental phase transitions [7-9], signal transduction [10], abiotic [11,12] and biotic [13] stress responses, and phytohormone regulation [14]. The plant miRNAs were first discovered in Arabidopsis thaliana in 2002 [15-17]. Since then, considerable efforts have been exerted on identifying (e.g., [18-21]) and functionally annotating (e.g., [22-24]) the plant miRNAs. To date, 6,992 precursor miRNAs and 8,496 mature miRNAs from 73 plant species have been deposited in public miRNA database (miRBase 21.0, http://www.mirbase.org). Since miRNAs are highly conserved in plants, this database provides a useful platform for predicating miRNAs in other plants based on sequences homology.

The miRNAs have been found to be involved in regulating versatile biological processes, in particular, their role in regulating flower development has drawn much interest in the recent decades. For example, miR172 is found to act as a translational repressor of the APETALA2 genes in the $\mathrm{ABC}$ model of flower development. The overexpression of miRNA172 results in early flowering and disrupts the floral organ differentiation in A. thaliana [25]. Besides miR172, miR164 in $A$. thaliana is found to target the NAC transcription factors, including CUP-SHAPED COTYLEDON1 (CUC1) and CUC2, which are redundantly required for the formation of boundaries that separate organ primordia; in particular, miR164c controls the number of flower petals by regulating the accumulation of $C U C 1$ and $C U C 2$ transcripts 
[26]. More recently, miR156 is found to target the SPL genes, which regulate floral transitions by modulating the expression of the key floral-promoting genes in A. thaliana [27] and maize [28]. Furthermore, miR319, miR159, miR164, and miR167 are found to have response for specifying particular cell types in the later stages of floral development [29-32].

Sex determination mechanism in plants has long been of considerable interest. Recent studies reveal that miRNAs also play an important role in determining the sexes of plants. The miRNAs regulating carpel and stamen development can ultimately lead to the production of unisexual or bisexual flowers. For instance, the tasselseed 4 miRNA (miR172) is found to be involved in the sex determination of the male inflorescence in maize [33,34], which inhibits the translation of the IDS1 transcript in the male florets. In the ts 4 or ids 1 mutants, female organs develop in the male inflorescence. Very recently, a study reported that a Yspecific sex-determinant candidate gene (OGI) was detected in persimmons (Diospyros lotus). OGI encodes a small RNA that targets and represses the expression of the feminizing male growth inhibitor (MeGI) gene in male flowers [35]. The study reveals a mechanism that an autosomal feminizing gene suppressed by a Y-chromosome-encoded small RNA triggers the plant dioecy in which male is the heterogametic gender (XY).

Willows (Salix) are dioecious plants. The alternate sexes of willows bear morphologically different male or female catkins. A variety of studies presented reliable evidences that a single locus governed the sex determination in different willow species [36-40]. To date, all the mapping studies have confirmed that the female willows are the heterogametic gender, while the males are the homogametic sex, suggesting a ZW sex determination system in willows, and the gender locus is consistently mapped to the centromeric region on willow's chromosome XV [38-40]. With an international cooperative effort, the genome of $S$. suchowensis has been sequenced and the sequence scaffolds have been mapped along the 19 haploid chromosomes of willow [39, 41]. However, sequence assembly at the gender locus is fairly poor due to the high repetitive sequences in the centromeric region. In a previous study, we also analyzed the differentially expressed genes based on transcriptome sequencing of flower buds of the alternate sexes and detected 33 differentially expressed genes located on the incipient sex chromosome of Salicaceae [42]. Thus far, no obvious gender determinant has been identified based on the aforementioned studies. In consideration of the role of miRNAs in determining the sexes of plants, in this study, we predict the microRNAs and their targets from transcriptome sequences derived from male and female flowers of $S$. suchowensis. Our goal is to detect the candidate sex determinant of willow following the new perspective on role of miRNAs in sex determination of plants.

\section{Methods}

2.1. Sequence Resources. Reference miRNAs were downloaded from the publicly available miRBase database (http:// www.mirbase.org/, Release 21, June 2014), which included
8,496 known mature miRNAs derived from 73 plant species. Duplicates in this database were manually culled out by an inhouse multiple sequence alignment Perl script. The remaining unique mature miRNAs were used for homology search in the subsequent analysis. Transcriptome data were separately derived from flower buds of the alternate sexes of willow, which were deposit in the EMBL Nucleotide Archive (ENA, http://www.ebi.ac.uk/ena), with an ArrayExpress accession number of E-MTAB-1445. The transcriptome data totally contained 1,201,931 expressed sequence tags (ESTs) from both sexes, with an average length of $389 \mathrm{bp}$ and a total length of $467.96 \mathrm{Mb}$. Among them, 629,854 and 572,089 ESTs were from the female and male flower buds, respectively.

2.2. Bioinformatic Prediction of Potential miRNAs. Prediction of miRNAs was performed based on sequence homology between the transcriptome sequences of willows and the screened miRNAs from the miRBase database following the method described by Zhang et al. [43]. Firstly, the unique mature miRNAs were aligned to the transcriptome sequences of the alternate sexes of willows by the Bowtie alignment toolkit [44]. In this analysis, the EST sequences with less than three nucleotide mismatches were chosen as candidate miRNA precursors. Subsequently, the candidate precursors were aligned to the NCBI nonredundant protein database (https://www.ncbi.nlm.nih.gov/) and RNA database Rfam (http://rfam.xfam.org/) to eliminate protein-coding sequences and noncoding RNAs (e.g., tRNA, rRNA, snRNA, or snoRNA) by BLAST search [45]. Finally, the remaining candidates from above analyses were further assessed by RNAfold from the ViennaRNA Package with default parameters [46], which produced parameters for examining the secondary hairpin structure, the Dicer cleavage site, and the minimum free energy (MFE) of the candidate sequences. Based on these parameters, potential miRNAs were manually identified according to the criteria for predicating plant miRNAs as described by Zhang et al. [43].

2.3. Analyzing the miRNA Targets. Bioinformatic prediction of the miRNA targets was conducted based on sequence complementarity by using the psRNATarget program with default parameters (http://plantgrn.noble.org/psRNATarget/) [47]. Annotation of the putative targets was performed by aligning the target sequences to NCBI nonredundant protein database (https://www.ncbi.nlm.nih.gov/) by BLAST searches. The annotated targets were assigned with confined GO terms in the UniProt database [48] and plotted by using the online analytical tool of WEGO (http:// wego.genomics.org.cn/cgi-bin/wego/index.pl) [49]. The annotation information was used to retrieve keywords to identify genes related to sex determination and flowering.

The expression levels of miRNA targets in the male and female flowers were evaluated based on the number of reads captured from sequences of the targets. Sequence reads from flowers of the alternate sexes were separately aligned to the target sequences by using BWA (mismatch $\leq$ $2 \mathrm{bp}$, other parameters as default) [50]. And then, the SAM output from BWA was converted into a binary file (BAM) by using Samtools [51]. The number of mapped reads for each 
TABLE 1: Potential miRNAs identified in female flower buds of S. suchowensis.

\begin{tabular}{|c|c|c|c|c|c|}
\hline miRNA & miRNA sequence $\left(5^{\prime}-3^{\prime}\right)$ & LM (nt) & Arm & Pre-miR location & LP (nt) \\
\hline ssu-miR162a & UCGAUAAACCUCUGCAUCCAG & 21 & $3^{\prime}$ & seq459792:217..303:+ & 86 \\
\hline ssu-miR162b & UCGAUAAACCUCUGCAUCCAG & 21 & $3^{\prime}$ & seq351845:128..215:- & 87 \\
\hline ssu-miR162c & UCGAUAAACCUCUGCAUCCAG & 21 & $3^{\prime}$ & seq365695:26..113:+ & 87 \\
\hline ssu-miR166 & GGAAUGUUGUCUGGCUCGAGG & 21 & $5^{\prime}$ & seq576601:133..255:+ & 122 \\
\hline ssu-miR169 & CAGCCAAGGAUGACUUGCCGG & 21 & $3^{\prime}$ & $\begin{array}{l}\text { seq292249:428..483:- } \\
\text { seq292249:488..543:- }\end{array}$ & 55 \\
\hline ssu-miR390 & CGCUAUCCAUCCUGAGUUUCA & 21 & $3^{\prime}$ & seq242088:345..447:- & 102 \\
\hline ssu-miR172a & AGAAUCCUGAUGAUGCUGCAG & 21 & $5^{\prime}$ & $\begin{array}{l}\text { seq288542:116..254:+ } \\
\text { seq263722:286..424:+ } \\
\text { seq600570:208..346:- }\end{array}$ & 138 \\
\hline ssu-miR472a & UUUUCCCAACUCCACCCAUCCC & 22 & $3^{\prime}$ & seq62383:374..435:- & 61 \\
\hline ssu-miR472b & UUUUCCCAACUCCACCCAUCCC & 22 & $3^{\prime}$ & seq355906:377..445:- & 68 \\
\hline ssu-miR1448 & CUUUCCAACGCCUCCCAUAC & 20 & $3^{\prime}$ & seq140125:105..174:+ & 69 \\
\hline ssu-miR6423 & CCGCUGUCGCCACUAUCUUCCU & 22 & $5^{\prime}$ & $\begin{array}{c}\text { seq431318:268..328:+ } \\
\text { seq593886:92..152:+ }\end{array}$ & 60 \\
\hline
\end{tabular}

LM: length of miRNA; Arm: location of mature miRNAs on secondary stem-loop structures of pre-miRNA sequences; Pre-miR location: miRNA precursor's location in ESTs of willow female flower buds; LP: length of pre-miRNA.

target gene was counted. Normalization of the mapped reads was estimated as transcripts per million (TPM) [52]. The heatmap for sex-specific expression profiling of each target was generated based on the $\log 2$ TPM values by using $R$ package [53].

Since the gender locus of willow was confirmedly mapped in the centromeric region of chromosome XV, we demonstrated the chromosomal location of each target by aligning them to the genome sequence assemblies of S. suchowensis with an in-house Perl script.

\section{Results and Discussion}

3.1. Bioinformatic Predication of miRNAs. A total of 8,496 mature plant miRNAs were downloaded from the miRBase database. After eliminating the duplicate miRNA sequences, we obtained 4,803 nonredundant reference miRNAs, which were used to search for homologs in the EST sequences derived from flower buds of alternate sexes. This analysis yielded 356 and 527 ESTs that were perfect or nearperfect matches for the reference miRNA sequences from the female and from the male, respectively. We then culled out the protein-coding sequences and noncoding RNAs such as tRNA, rRNA, snRNA, and snoRNA from the matched ESTs, and 117 ESTs from the female and 183 ESTs from the male remained for secondary structures analysis. Whether a stem-loop hairpin present in secondary structure of the sequences is a common feature of miRNA precursors, the ESTs failed to meet this criterion are eliminated. Finally, 11 miRNAs representing eight miRNAs families were identified in the female, including three miR162 members, two miR472 members, and one member for each of the remaining six miRNA families (Table 1), whereas, in the male flower buds, we identified 23 miRNAs from 11 miRNA families, including four miR6423 members, three miR167 members, and one or two members for each of the remaining families (Table 2).
Similar to animal miRNAs, plant miRNAs are normally in lengths of 20-24 bp and are processed from longer precursor transcripts [54]. The lengths of miRNAs identified in this study were in range of 20-22 bp, and the majority of them (18 miRNAs) were in length of $21 \mathrm{bp}$. In animals, the premiRNAs typically contain 70-80 nucleotides. By contrast, plant miRNA precursors are more variable in structure and size, ranging from 50 to 350 nucleotides or even longer. In some plants, the miRNA precursors were found to exceed 1000 nucleotides [54, 55]. In this study, the lengths of miRNA precursors were in range of 51-138 nucleotides, with an average of 85 nucleotides. All the mature ssu-miRNA sequences were located on the stem-arm of the stem-loop hairpin secondary structure of the potential pre-miRNAs. However, the location of mature miRNAs varied in different pre-miRNAs, such as ssu-miR156, ssu-miR160, ssu-miR166, ssu-miR172, and ssu-miR6423, the mature miRNA sequences located at the $5^{\prime}$ arm of the stem-loop hairpin. By contrast, the mature miRNA sequences in the remaining pre-miRNAs present at the $3^{\prime}$ arm of the stem-loop hairpin (Tables 1 and 2 ). All the pre-miRNAs identified in this study form stable stem-loop hairpins in their secondary structure (Figure 1).

3.2. Compare the miRNAs Identified in the Alternate Sexes. For the identified ssu-miRNAs (Table 3), they were classified into eight miRNA families in the female and classified into 11 miRNA families in the male. Five of the miRNA families were found to be common in the female and male flowers (i.e., ssumiR162, ssu-miR169, ssu-miR472a, ssu-miR1448, and ssumiR6243). By contrast, there were specific miRNA families (ssu-miR166, ssu-miR390) and miRNAs (ssu-miR172a) that were only detected in the female, and there were specific miRNA families (including ssu-miR156, ssu-miR160, ssumiR167, ssu-miR396, ssu-miR7841, and ssu-miR396) and miRNAs (including ssu-miR162d, ssu-miR472c, and ssu$\mathrm{miR} 172 \mathrm{~b} / \mathrm{c}$ ) that were only detected in the male. 
TABle 2: Potential miRNAs identified in male flower buds of S. suchowensis.

\begin{tabular}{|c|c|c|c|c|c|}
\hline miRNA & miRNA sequence $\left(5^{\prime}-3^{\prime}\right)$ & $\mathrm{LM}(\mathrm{nt})$ & Arm & Pre-miR location & $\mathrm{LP}(\mathrm{nt})$ \\
\hline ssu-miR156a & UGACAGAAGAUAGAGAGCAC & 20 & $5^{\prime}$ & seq517259:84..165:+ & 81 \\
\hline ssu-miR156b & UGACAGAAGAUAGAGAGCAC & 20 & $5^{\prime}$ & seq325710:29..80:- & 51 \\
\hline ssu-miR160 & UGCCUGGCUCCCUGUAUGCC & 20 & $5^{\prime}$ & seq145824:369..444:- & 75 \\
\hline ssu-miR167a & AGAUCAUGUGGCAGUUUCACC & 21 & $3^{\prime}$ & $\begin{array}{l}\text { seq137705:68..140:+ } \\
\text { seq383115:96..168:+ }\end{array}$ & 72 \\
\hline ssu-miR167b & AGAUCAUGUGGCAGUUUCACC & 21 & $3^{\prime}$ & seq450474:97..169:+ & 72 \\
\hline ssu-miR167c & AGAUCAUGUGGCAGUUUCACC & 21 & $3^{\prime}$ & $\begin{array}{l}\text { seq460555:421..495:- } \\
\text { seq460555:481..555:- }\end{array}$ & 74 \\
\hline ssu-miR162a & UCGAUAAACCUCUGCAUCCAG & 21 & $3^{\prime}$ & seq430376:217..303:+ & 86 \\
\hline ssu-miR162d & UGGAGGCAGCGGUUCAUCGAUC & 22 & $5^{\prime}$ & seq344645:410..500:+ & 90 \\
\hline ssu-miR169a & CAGCCAAGGAUGACUUGCCGG & 21 & $5^{\prime}$ & seq159785:89..210:- & 121 \\
\hline ssu-miR169b & CAGCCAAGGAUGACUUGCCGG & 21 & $5^{\prime}$ & $\begin{array}{c}\text { seq167083:107..214:+ } \\
\text { seq166207:31..138:+ }\end{array}$ & 107 \\
\hline ssu-miR172b & GCAGCACCAUCAAGAUUCACA & 21 & $5^{\prime}$ & seq555414:28..136:+ & 108 \\
\hline ssu-miR172c & GCAGCACCAUCAAGAUUCACA & 21 & $5^{\prime}$ & seq562308:14..122:- & 108 \\
\hline ssu-miR396a & UUCCACAGCUUUCUUGAACUG & 21 & $3^{\prime}$ & seq114772:89..196:+ & 107 \\
\hline ssu-miR396b & UUCCACAGCUUUCUUGAACUG & 21 & $3^{\prime}$ & seq307347:218..305:- & 87 \\
\hline ssu-miR472a & UUUUCCCAACUCCACCCAUCCC & 22 & $3^{\prime}$ & seq467610:63..125:+ & 62 \\
\hline ssu-miR472c & UCUUGCCUACUCCUCCCAUUCC & 22 & $3^{\prime}$ & seq106361:38..109:+ & 71 \\
\hline ssu-miR1448a & CUUUCCAACGCCUCCCAUAC & 20 & $3^{\prime}$ & seq106361:219..287:+ & 68 \\
\hline ssu-miR1448b & CUUUCCAACGCCUCCCAUAC & 20 & $3^{\prime}$ & $\begin{array}{l}\text { seq83628:392..461:- } \\
\text { seq83628:422..491:- }\end{array}$ & 69 \\
\hline ssu-miR7841 & GGGGGUUGCUGUCAAGCAUAA & 21 & $3^{\prime}$ & seq306990:54..154:- & 100 \\
\hline ssu-miR6423a & CCGCUGUCGCCACUAUCUUCCU & 22 & $5^{\prime}$ & seq268392:35..125:+ & 90 \\
\hline ssu-miR6423b & CCGCUGUCGCCACUAUCUUCCU & 22 & $5^{\prime}$ & seq228745:13..103:+ & 90 \\
\hline ssu-miR6423c & CCGCUGUCGCCACUAUCUUCCU & 22 & $5^{\prime}$ & seq199601:0..96:+ & 96 \\
\hline ssu-miR6423d & CCGCUGUCGCCACUAUCUUCCU & 22 & $5^{\prime}$ & $\begin{array}{l}\text { seq100248:45..105:- } \\
\text { seq148917:45..105:- }\end{array}$ & 60 \\
\hline
\end{tabular}

LM: length of miRNA; Arm: location of mature miRNAs on secondary stem-loop structures of pre-miRNA sequences; Pre-miR location: miRNA precursor's location in ESTs of willow male flower buds; LP: length of pre-miRNA.

In the identified ssu-miRNAs, five miRNA families (i.e., ssu-miR169, ssu-miR162, ssu-miR166, ssu-miR156, and ssu-miR396) were found to have the same sequences as that in more than 10 plant species. Complete matches to ssu-miR169, ssu-miR396, ssu-miR162, ssu-miR156, and ssumiR166 sequences were detected in 58, 44, 35, 14, and 12 plant species, respectively (Table 3 ). The targets of these miRNA families were found to play important roles in flower development. For example, target of miR169 is a class- $\mathrm{C}$ gene that specify the identities of the reproductive organs during flower development in Petunia hybrida and Antirrhinum majus $[56,57]$, whereas miR156 is one of the most ancient miRNAs in perhaps all land plant lineages, which is a key regulator of vegetative phase change in Arabidopsis, maize, and rice [58]. Overexpression of miR156 results in prolonged expression of juvenile characters and extremely delayed flowering. Although most miRNAs are highly conserved in different plant species, specific miRNAs have been detected in Salicaceae species (Table 3). In this study, we also detected some Salicaceae-specific miRNAs (e.g., ssumiR6423, ssu-miR472, ssu-miR1448, and ssu-miR7841) in $S$. suchowensis.
3.3. Analyzing the Targets of the Identified miRNAs. MiRNAs generally regulate the expression of specific genes via mRNA cleavage or translational inhibition. Based on the perfect or near-perfect sequence complementarity between miRNAs and their target transcripts, 55 targets (Table S1 in Supplementary Material available online at https://doi.org/10.1155/2017/9614596) for eight miRNAs from female flower buds and 124 targets (Table S2) for 13 miRNAs from male flower buds were detected separately (Figure S1). It showed that ssu-miR396 had the most targets (35), followed by ssu-miR472a (20) and ssu-miR156 (19).

Based on the GO terms of the corresponding homolog in the UniProt database [48], 44 (80\%) and $96(77 \%)$ targets from female (Table S3) and male (Table S4) flower buds were assigned with a confined GO term, respectively. The functionally annotated target genes belonged to categories of biological process, cellular component, and molecular function. Functional classification of the annotated targets was shown in a WEGO chart (Figure S2). In general, number of targets that have fallen in different component groups showed a similar pattern between the male and female. However, targets from the male were significantly enriched in 


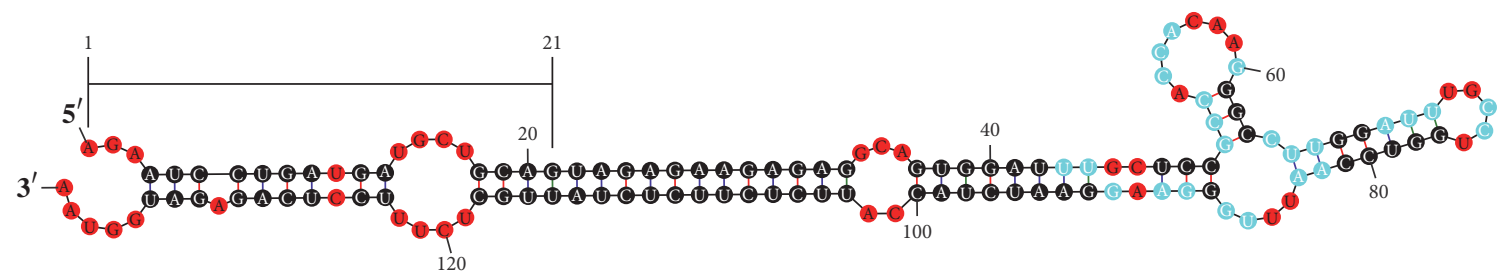

(a)

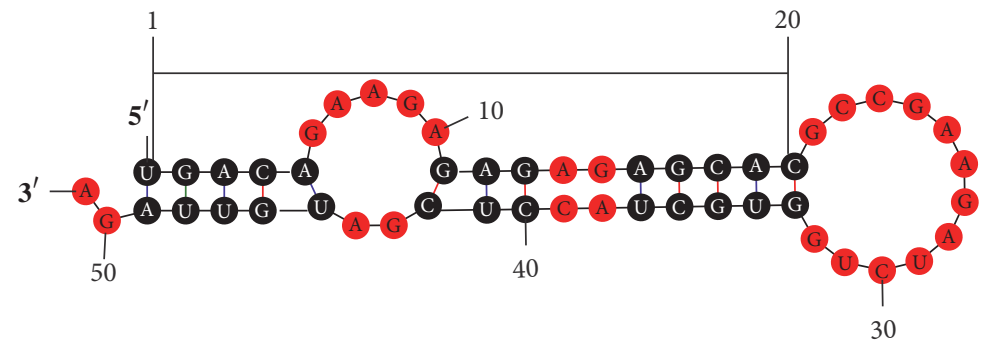

(b)

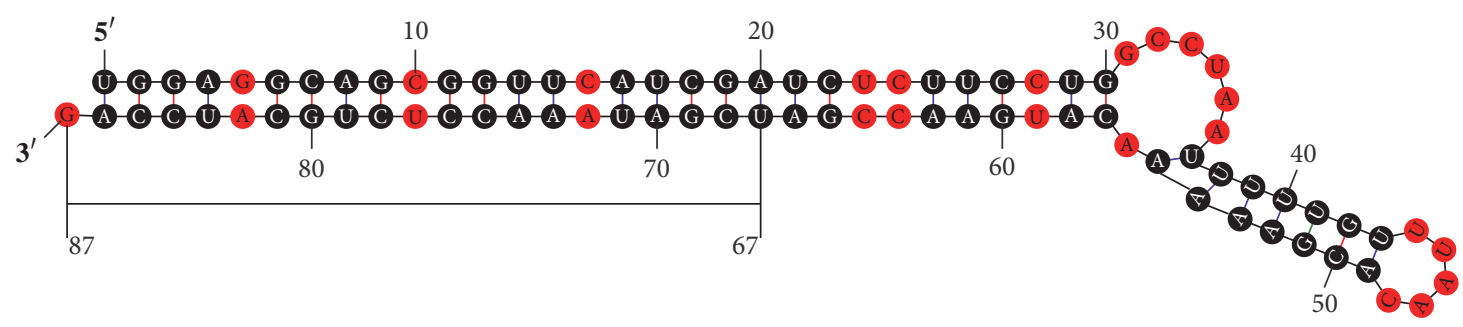

(c)

FIGURE 1: Three predicted stem-loop structures of pre-miRNAs identified in S. suchowensis. The mature miRNA is indicated with a black bar. (a) ssu-miR172a, whose precursor is in length of 138 bp. (b) ssu-miR156b, whose precursor is in length of 51 bp. (c) ssu-miR162b, whose precursor is in length of $87 \mathrm{bp}$.

TABLE 3: Summary of miRNAs identified in flower budsof the alternate sexes in S. suchowensis.

\begin{tabular}{|c|c|c|c|}
\hline Name & miRNA family & miRNA sequence $\left(5^{\prime}-3^{\prime}\right)$ & Number of species \\
\hline \multirow{5}{*}{ Common in female and male } & ssu-miR6423 & CCGCUGUCGCCACUAUCUUCCU & 1 \\
\hline & ssu-miR169 & CAGCCAAGGAUGACUUGCCGG & 58 \\
\hline & ssu-miR162 & UCGAUAAACCUCUGCAUCCAG & 35 \\
\hline & ssu-miR472a & UUUUCCCAACUCCACCCAUCCC & 1 \\
\hline & ssu-miR1448 & CUUUCCAACGCCUCCCAUAC & 1 \\
\hline \multirow{3}{*}{ Female-specific } & ssu-miR166 & GGAAUGUUGUCUGGCUCGAGG & 12 \\
\hline & ssu-miR390 & CGCUAUCCAUCCUGAGUUUCA & 5 \\
\hline & ssu-miR172a & AGAAUCCUGAUGAUGCUGCAG & 3 \\
\hline \multirow{8}{*}{ Male-specific } & ssu-miR156 & UGACAGAAGAUAGAGAGCAC & 14 \\
\hline & ssu-miR162d & UGGAGGCAGCGGUUCAUCGAUC & 3 \\
\hline & ssu-miR160 & UGCCUGGCUCCCUGUAUGCC & 6 \\
\hline & ssu-miR7841 & GGGGGUUGCUGUCAAGCAUAA & 1 \\
\hline & ssu-miR167 & AGAUCAUGUGGCAGUUUCACC & 4 \\
\hline & ssu-miR396 & UUCCACAGCUUUCUUGAACUG & 44 \\
\hline & ssu-miR472c & UCUUGCCUACUCCUCCCAUUCC & 1 \\
\hline & ssu-miR172b/c & GCAGCACCAUCAAGAUUCACA & 2 \\
\hline
\end{tabular}

Number of species indicates in how many plant species the homologous miRNA was detected. 

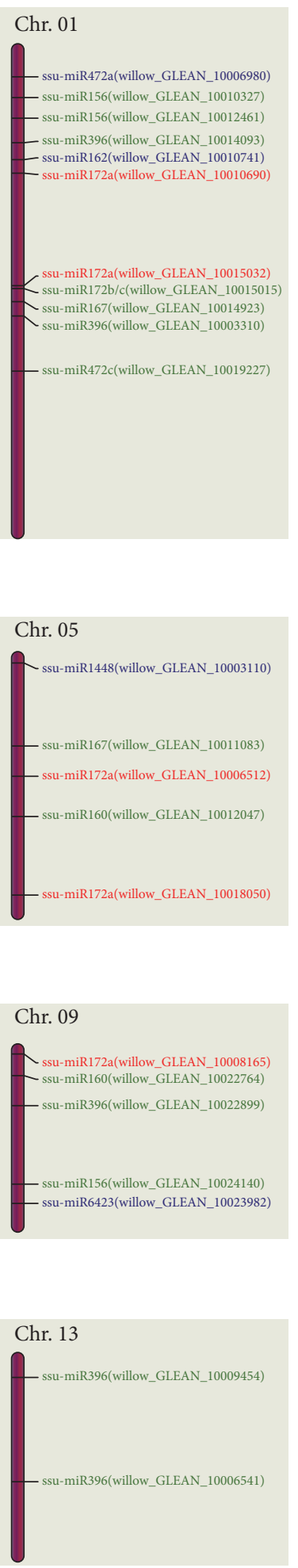

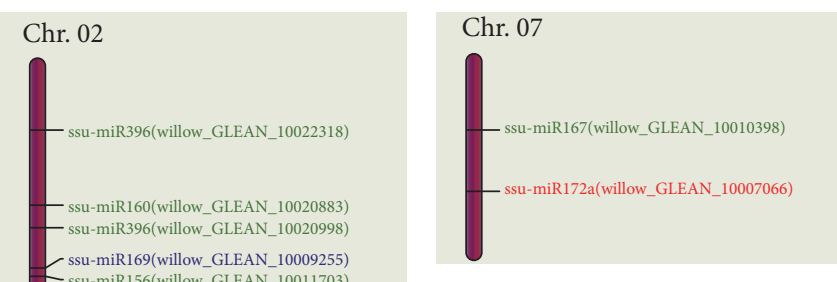

\section{Chr. 14}

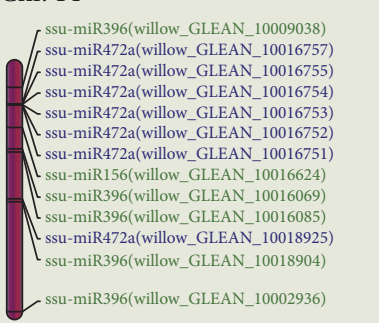

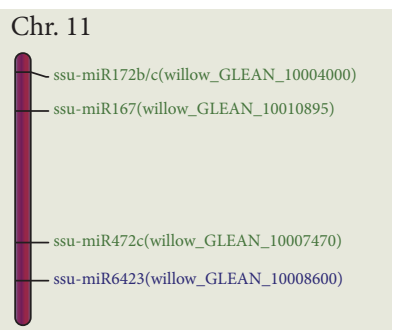

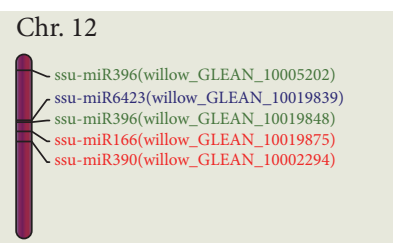

\section{Chr. 15}

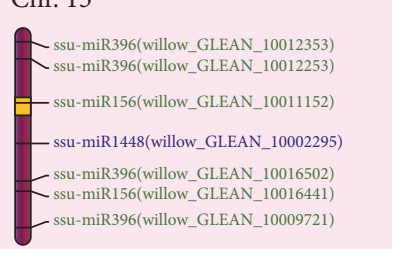

Chr. 17
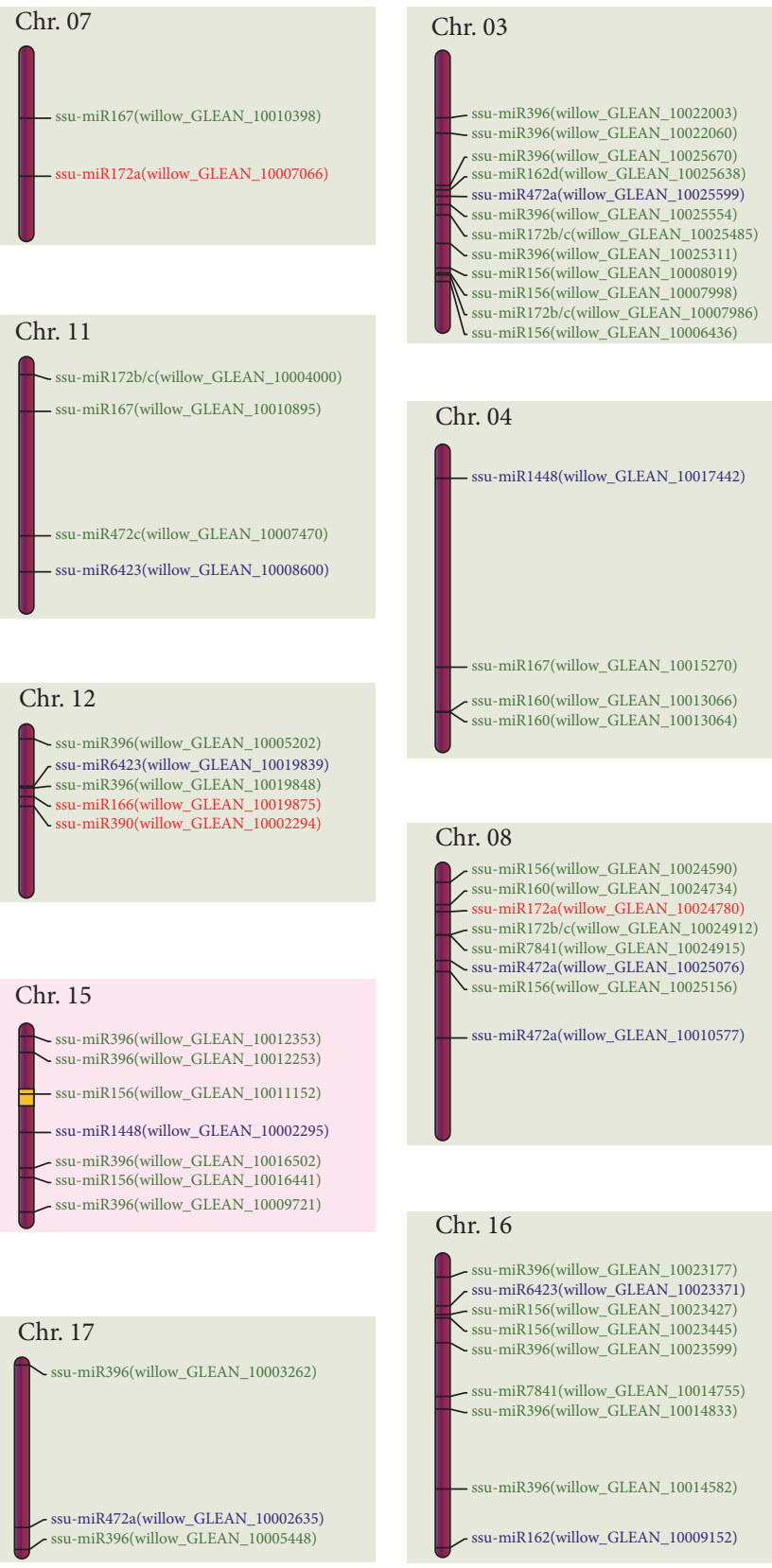

Chr. 08

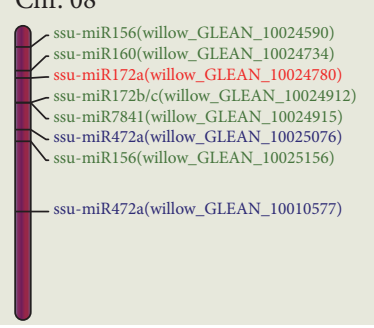

Chr. 16

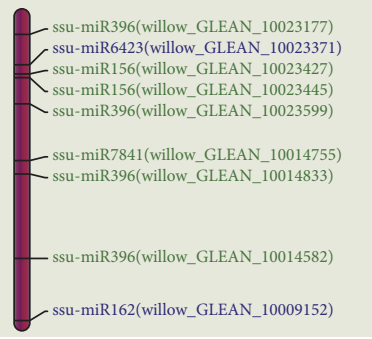

Chr. 18

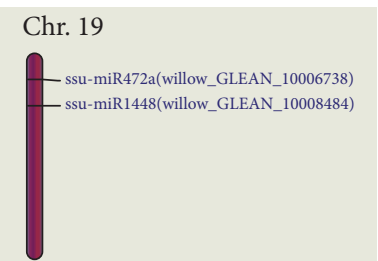

FIGURE 2: Distribution of miRNA targets on 19 chromosomes of S. suchowensis. Thirty target genes regulated by miRNAs commonly presented in both the female and the male flower buds are shown with blue, whereas 17 and 84 genes regulated by miRNAs presented exclusively in the female or the male are shown with red and green, respectively. In addition, the yellow block in chromosome XV represents gender locus in sex chromosome of S. suchowensis. 
some of the component groups, such as in component groups of envelope, antioxidant, enzyme regulator activity, molecular transducer, and cellular component biogenesis.

In order to gain more information for characterization of functional miRNAs involved in sex-specific flower development, we analyzed the expression profiles of the detected miRNA targets in male and female flower buds separately. Totally, 123 targets were found to be expressed in the male or female flower buds. Among them, only 8 targets are expressed in the female, only 8 targets are expressed in the male, and 107 targets are expressed in both male and female flower buds (Figure S3, Table S5). According to the TPM values, a negative correlation was observed between the expression of miRNAs and that of their targets. For some extremes, ssumiR166, ssu-miR390, and ssu-miR172a were detected only in the female flower buds. Consequently, their targets had significantly lower expression level in female than in male flowers. In cases of ssu-miR156, ssu-miR162d, ssu-miR160, ssu-miR167, ssu-miR472c, ssu-miR172b/c, and ssu-miR7841, these miRNAs were only detected in the male, and their targets were found to be expressed significantly less in male than in female flowers. The negative correlation between the expression levels of miRNAs and those of their targets is in accordance with the gene silencing function of miRNAs.

\subsection{Mapping the miRNA Targets along Chromosomal Assem-} blies of S. suchowensis. Based on the chromosomal assemblies established by Hou et al. [39], we plotted the distribution of the predicted targets along each chromosome in the willow genome. Totally, 47 targets detected in the female and 114 targets detected in the male were mapped onto the 19 haploid chromosomes in the genome of S. suchowensis (Figure 2, Table S6). Among them, 30 targets were regulated by miRNAs commonly presented in both the female and the male flower buds (in blue), 17 targets were regulated by female-specific miRNAs (in red), and 84 targets were regulated by malespecific miRNAs (in green). On many of the chromosomes, targets regulated by male-specific miRNAs were denser than those regulated by female-specific miRNAs.

The previous mapping study has confined the gender locus in a $742 \mathrm{~Kb}$ centromeric region on chromosome $\mathrm{XV}$ (the yellow block, Figure 2) [39]. It is notable that a target (willow_GLEAN_10011152) regulated by a male-specific miRNA (ssu-miR156) is located in the gender determination region. This target is expressed fairly highly in female flower buds, but no expression of it is observed in male flower buds (TPM: 576.4 versus 0). Furthermore, functional annotation of this target indicates that it is a transcription factor involved in the control of early flower development. Dedicated lab experiments need to be conducted to learn the biological role of these genes and their regulatory miRNA in willow sex determination. Salicaceae is a family of dioecious plants and possess the ZW sex determination system in which the female is the heterogametic gender $[39,59,60]$. Species with ZW sex determination system are relatively rare in high plants; thus willow provides a desirable system to learn the genetic mechanism underlying the ZW sex determination in plants. Although tremendous endeavor has been made to identify the sex-determining gene in Salicaceae species [38-40], no obvious candidate gene has been detected thus far. In this study, we explore the candidate in terms of miRNA perspective and detected an obvious candidate associated with flower development and sex differentiation of willow. However, this finding is discovered only based on the bioinformatic analyses; it need to be validated with experimental proof. Nevertheless, we identify a candidate sex determinant in willow by using bioinformatic analytical tools, and this finding is worthy of being explored by dedicated experimental efforts in future studies.

\section{Conclusions}

miRNAs are regulatory factors with important functions in diverse processes related to plant growth, development, morphogenesis, and stress responses. In particular, they are found to play important roles in regulating flower development, as well as sex differentiation of high plants. We analyzed the transcriptome sequences derived from the flower buds of the alternate sex of willow, focusing on predicating the putative miRNAs and their targets. Together with the genome sequence and mapping results of gender locus in willow, this work enables us to identify a candidate of sex determinant, which is a transcription factor involved in early flower development and the expression of it is only observed in female flower buds. Although we need to conduct a series of experimental studies to validate this bioinformatic finding, and the detailed mechanism on how the candidate regulates sex determination remains largely unknown; our work provides a valuable bioinformatic clue for further exploration to uncover the genetic mechanism underlying sex determination of willows, in which gender occurred through the ZW sex determination system.

\section{Conflicts of Interest}

The authors declare that they have no conflicts of interest.

\section{Acknowledgments}

The work is supported by the National Key Research and Development Plan of China (2016YFD0600101) and the National Natural Science Foundation of China (31561123001). It is also enabled by the Innovative Research Team Program of the Educational Department of China and the PAPD (Priority Academic Program Development) program at Nanjing Forestry University.

\section{References}

[1] D. P. Bartel, "MicroRNAs: target recognition and regulatory functions," Cell, vol. 136, no. 2, pp. 215-233, 2009.

[2] A. Eulalio, E. Huntzinger, and E. Izaurralde, "Getting to the root of miRNA-mediated gene silencing," Cell, vol. 132, no. 1, pp. 914, 2008.

[3] S. M. Elbashir, W. Lendeckel, and T. Tuschl, "RNA interference is mediated by 21- and 22-nucleotide RNAs," Genes and Development, vol. 15, no. 2, pp. 188-200, 2001. 
[4] M. J. Axtell and D. P. Bartel, "Antiquity of microRNAs and their targets in land plants," The Plant Cell, vol. 17, no. 6, pp. 16581673, 2005.

[5] O. Voinnet, "Origin, biogenesis, and activity of plant microRNAs," Cell, vol. 136, no. 4, pp. 669-687, 2009.

[6] M. J. Axtell, J. A. Snyder, and D. P. Bartel, "Common functions for diverse small RNAs of land plants," Plant Cell, vol. 19, no. 6, pp. 1750-1769, 2007.

[7] S. Jasinski, A. C. M. Vialette-Guiraud, and C. P. Scutt, "The evolutionary-developmental analysis of plant microRNAs," Philosophical Transactions of the Royal Society B: Biological Sciences, vol. 365, no. 1539, pp. 469-476, 2010.

[8] O. Valdés-López, C. Arenas-Huertero, M. Ramírez et al., "Essential role of MYB transcription factor: PvPHR1 and microRNA: PvmiR399 in phosphorus-deficiency signalling in common bean roots," Plant, Cell and Environment, vol. 31, no. 12, pp. 1834-1843, 2008.

[9] H. S. Guo, Q. Xie, J. F. Fei, and N. H. Chua, "MicroRNA directs mRNA cleavage of the transcription factor NAC1 to downregulate auxin signals for Arabidopsis lateral root development," Plant Cell, vol. 17, no. 5, pp. 1376-1386, 2005.

[10] J.-Y. Gou, F. F. Felippes, C.-J. Liu, D. Weigel, and J.-W. Wang, "Negative regulation of anthocyanin biosynthesis in arabidopsis by a miR156-targeted SPL transcription factor," Plant Cell, vol. 23, no. 4, pp. 1512-1522, 2011.

[11] T. Wang, L. Chen, M. Zhao, Q. Tian, and W.-H. Zhang, "Identification of drought-responsive microRNAs in Medicago truncatula by genome-wide high-throughput sequencing," $B M C$ Genomics, vol. 12, article 367, 2011.

[12] Y. Qin, Z. Duan, X. Xia, and W. Yin, "Expression profiles of precursor and mature microRNAs under dehydration and high salinity shock in Populus euphratica," Plant Cell Reports, vol. 30, no. 10, pp. 1893-1907, 2011.

[13] L. Chen, Y. Ren, Y. Zhang, J. Xu, Z. Zhang, and Y. Wang, "Genome-wide profiling of novel and conserved Populus microRNAs involved in pathogen stress response by deep sequencing," Planta, vol. 235, no. 5, pp. 873-883, 2012.

[14] K. Aung, S.-I. Lin, C.-C. Wu, Y.-T. Huang, C.-L. Su, and T.J. Chiou, "pho2, a phosphate overaccumulator, is caused by a nonsense mutation in a microRNA399 target gene," Plant Physiology, vol. 141, no. 3, pp. 1000-1011, 2006.

[15] W. Park, J. Li, R. Song, J. Messing, and X. Chen, "CARPEL FACTORY, a Dicer homolog, and HEN1, a novel protein, act in microRNA metabolism in Arabidopsis thaliana," Current Biology, vol. 12, no. 17, pp. 1484-1495, 2002.

[16] B. J. Reinhart, E. G. Weinstein, M. W. Rhoades, B. Bartel, and D. P. Bartel, "MicroRNAs in plants," Genes and Development, vol. 16, no. 13, pp. 1616-1626, 2002.

[17] C. Llave, Z. Xie, K. D. Kasschau, and J. C. Carrington, "Cleavage of Scarecrow-like mRNA targets directed by a class of Arabidopsis miRNA," Science, vol. 297, no. 5589, pp. 2053-2056, 2002.

[18] X.-J. Wang, J. L. Reyes, N.-H. Chua, and T. Gaasterland, "Prediction and identification of Arabidopsis thaliana microRNAs and their mRNA targets," Genome Biology, vol. 5, no. 9, p. R65, 2004.

[19] A. Barakat, P. K. Wall, S. DiLoreto, C. W. dePamphilis, and J. E. Carlson, "Conservation and divergence of microRNAs in Populus," BMC Genomics, vol. 8, article 481, 2007.

[20] R. Sunkar, X. Zhou, Y. Zheng, W. Zhang, and J. Zhu, "Identification of novel and candidate miRNAs in rice by high throughput sequencing," BMC Plant Biology, vol. 8, article 25, 2008.
[21] J. Zhang, R. Zeng, J. Chen, X. Liu, and Q. Liao, "Identification of conserved microRNAs and their targets from Solanum lycopersicum Mill," Gene, vol. 423, no. 1, pp. 1-7, 2008.

[22] R. Sunkar, Y. F. Li, and G. Jagadeeswaran, "Functions of microRNAs in plant stress responses," Trends in Plant Science, vol. 17, no. 4, pp. 196-203, 2012.

[23] B. C. Meyers, M. J. Axtell, B. Bartel et al., "Criteria for annotation of plant microRNAs," Plant Cell, vol. 20, no. 12, pp. 3186-3190, 2008.

[24] B. Zhang, X. Pan, C. H. Cannon, G. P. Cobb, and T. A. Anderson, "Conservation and divergence of plant microRNA genes," Plant Journal, vol. 46, no. 2, pp. 243-259, 2006.

[25] M. J. Aukerman and H. Sakai, "Regulation of flowering time and floral organ identity by a microRNA and its APETALA2like target genes," The Plant Cell, vol. 15, no. 11, pp. 2730-2741, 2003.

[26] C. C. Baker, P. Sieber, F. Wellmer, and E. M. Meyerowitz, "The early extra petals1 mutant uncovers a role for microRNA miR164c in regulating petal number in Arabidopsis," Current Biology, vol. 15, no. 4, pp. 303-315, 2005.

[27] J.-W. Wang, B. Czech, and D. Weigel, "miR156-regulated SPL transcription factors define an endogenous flowering pathway in arabidopsis thaliana," Cell, vol. 138, no. 4, pp. 738-749, 2009.

[28] J. F. Hultquist and J. E. Dorweiler, "Feminized tassels of maize mop1 and ts1 mutants exhibit altered levels of miR156 and specific SBP-box genes," Planta, vol. 229, no. 1, pp. 99-113, 2008.

[29] A. Nag, S. King, and T. Jack, "miR319a targeting of TCP4 is critical for petal growth and development in Arabidopsis," Proceedings of the National Academy of Sciences of the United States of America, vol. 106, no. 52, pp. 22534-22539, 2009.

[30] R. S. Allen, J. Li, M. I. Stahle, A. Dubroué, F. Gubler, and A. A. Millar, "Genetic analysis reveals functional redundancy and the major target genes of the Arabidopsis miR159 family," Proceedings of the National Academy of Sciences of the United States of America, vol. 104, no. 41, pp. 16371-16376, 2007.

[31] P. Sieber, F. Wellmer, J. Gheyselinck, J. L. Riechmann, and E. M. Meyerowitz, "Redundancy and specialization among plant microRNAs: Role of the MIR164 family in developmental robustness," Development, vol. 134, no. 6, pp. 1051-1060, 2007.

[32] M.-F. Wu, Q. Tian, and J. W. Reed, "Arabidopis microRNA 167 controls patterns of ARF6 and ARF8 expression, and regulates both female and male reproduction," Development, vol. 133, no. 21, pp. 4211-4218, 2006.

[33] G. Chuck, R. Meeley, E. Irish, H. Sakai, and S. Hake, "The maize tasselseed 4 microRNA controls sex determination and meristem cell fate by targeting Tasselseed6/indeterminate spikelet1," Nature Genetics, vol. 39, no. 12, pp. 1517-1521, 2007.

[34] J. A. Banks, "MicroRNA, sex determination and floral meristem determinacy in maize," Genome Biology, vol. 9, no. 1, p. 204, 2008.

[35] T. Akagi, I. M. Henry, R. Tao, and L. Comai, "A Y-chromosomeencoded small RNA acts as a sex determinant in persimmons," Science, vol. 346, no. 6209, pp. 646-650, 2014.

[36] L. E. Gunter, G. T. Roberts, K. Lee, F. W. Larimer, and G. A. Tuskan, "The development of two flanking SCAR markers linked to a sex determination locus in Salix viminalis L," Journal of Heredity, vol. 94, no. 2, pp. 185-189, 2003.

[37] V. Semerikov, U. Lagercrantz, V. Tsarouhas, A. RönnbergWästljung, C. Alström-Rapaport, and M. Lascoux, "Genetic mapping of sex-linked markers in Salix viminalis L.," Heredity, vol. 91, no. 3, pp. 293-299, 2003. 
[38] P. Pucholt, A.-C. Rönnberg-Wästljung, and S. Berlin, "Single locus sex determination and female heterogamety in the basket willow (Salix viminalis L.)," Heredity, vol. 114, no. 6, pp. 575-583, 2015.

[39] J. Hou, N. Ye, D. Zhang et al., "Different autosomes evolved into sex chromosomes in the sister genera of Salix and Populus," Scientific Reports, vol. 5, article no. 9076, 2015.

[40] Y. Chen, T. Wang, L. Fang, X. Li, and T. Yin, "Confirmation of single-locus sex determination and female heterogamety in willow based on linkage analysis," PLoS ONE, vol. 11, no. 2, article e0147671, 2016.

[41] X. Dai, Q. Hu, Q. Cai et al., "The willow genome and divergent evolution from poplar after the common genome duplication," Cell Research, vol. 24, no. 10, pp. 1274-1277, 2014.

[42] J. Liu, T. Yin, N. Ye et al., "Transcriptome Analysis of the Differentially Expressed Genes in the Male and Female Shrub Willows (Salix suchowensis)," PLoS ONE, vol. 8, no. 4, Article ID e60181, 2013.

[43] B. H. Zhang, X. P. Pan, Q. L. Wang, G. P. Cobb, and T. A. Anderson, "Identification and characterization of new plant microRNAs using EST analysis," Cell Research, vol. 15, no. 5, pp. 336-360, 2005.

[44] B. Langmead, C. Trapnell, M. Pop, and S. L. Salzberg, "Ultrafast and memory-efficient alignment of short DNA sequences to the human genome," Genome Biology, vol. 10, no. 3, article R25, 2009.

[45] S. F. Altschul, W. Gish, W. Miller, E. W. Myers, and D. J. Lipman, "Basic local alignment search tool," Journal of Molecular Biology, vol. 215, no. 3, pp. 403-410, 1990.

[46] R. Lorenz, S. H. Bernhart, C. Höner Zu Siederdissen et al., "ViennaRNA Package 2.0," Algorithms for Molecular Biology, vol. 6, no. 1, article 26, 2011.

[47] X. Dai and P. X. Zhao, "PsRNATarget: a plant small RNA target analysis server," Nucleic Acids Research, vol. 39, no. 2, pp. W155W159, 2011.

[48] E. Camon, M. Magrane, D. Barrell et al., "The gene ontology annotation (GOA) database: sharing knowledge in uniprot with gene oncology," Nucleic Acids Research, vol. 32, pp. D262-D266, 2004.

[49] J. Ye, L. Fang, H. Zheng et al., "WEGO: a web tool for plotting GO annotations," Nucleic Acids Research, vol. 34, pp. W293W297, 2006.

[50] H. Li and R. Durbin, "Fast and accurate short read alignment with Burrows-Wheeler transform," Bioinformatics, vol. 25, no. 14, pp. 1754-1760, 2009.

[51] H. Li, B. Handsaker, A. Wysoker et al., "The sequence alignment/map format and SAMtools," Bioinformatics, vol. 25, no. 16, pp. 2078-2079, 2009.

[52] G. P. Wagner, K. Kin, and V. J. Lynch, "Measurement of mRNA abundance using RNA-seq data: RPKM measure is inconsistent among samples," Theory in Biosciences, vol. 131, no. 4, pp. 281285,2012

[53] R. Gentleman, "Reproducible research: a bioinformatics case study," Statistical Applications in Genetics and Molecular Biology, vol. 4, no. 1, article 2, 25 pages, 2005.

[54] J.-H. Jung, P. J. Seo, and C.-M. Park, "MicroRNA biogenesis and function in higher plants," Plant Biotechnology Reports, vol. 3, no. 2, pp. 111-126, 2009.

[55] B. Zhang, X. Pan, and T. A. Anderson, "Identification of 188 conserved maize microRNAs and their targets," FEBS Letters, vol. 580, no. 15, pp. 3753-3762, 2006.
[56] M. Cartolano, R. Castillo, N. Efremova et al., "A conserved microRNA module exerts homeotic control over Petunia hybrida and Antirrhinum majus floral organ identity," Nature Genetics, vol. 39, no. 7, pp. 901-905, 2007.

[57] X. Chen, "Small RNAs and their roles in plant development," Annual Review of Cell and Developmental Biology, vol. 25, pp. 21-44, 2009.

[58] R. S. Poethig, "Small RNAs and developmental timing in plants," Current Opinion in Genetics and Development, vol. 19, no. 4, pp. 374-378, 2009.

[59] T. Yin, S. P. DiFazio, L. E. Gunter et al., "Genome structure and emerging evidence of an incipient sex chromosome in Populus," Genome Research, vol. 18, no. 3, pp. 422-430, 2008.

[60] I. Paolucci, M. Gaudet, V. Jorge et al., "Genetic linkage maps of Populus alba L. and comparative mapping analysis of sex determination across Populus species," Tree Genetics and Genomes, vol. 6, no. 6, pp. 863-875, 2010. 

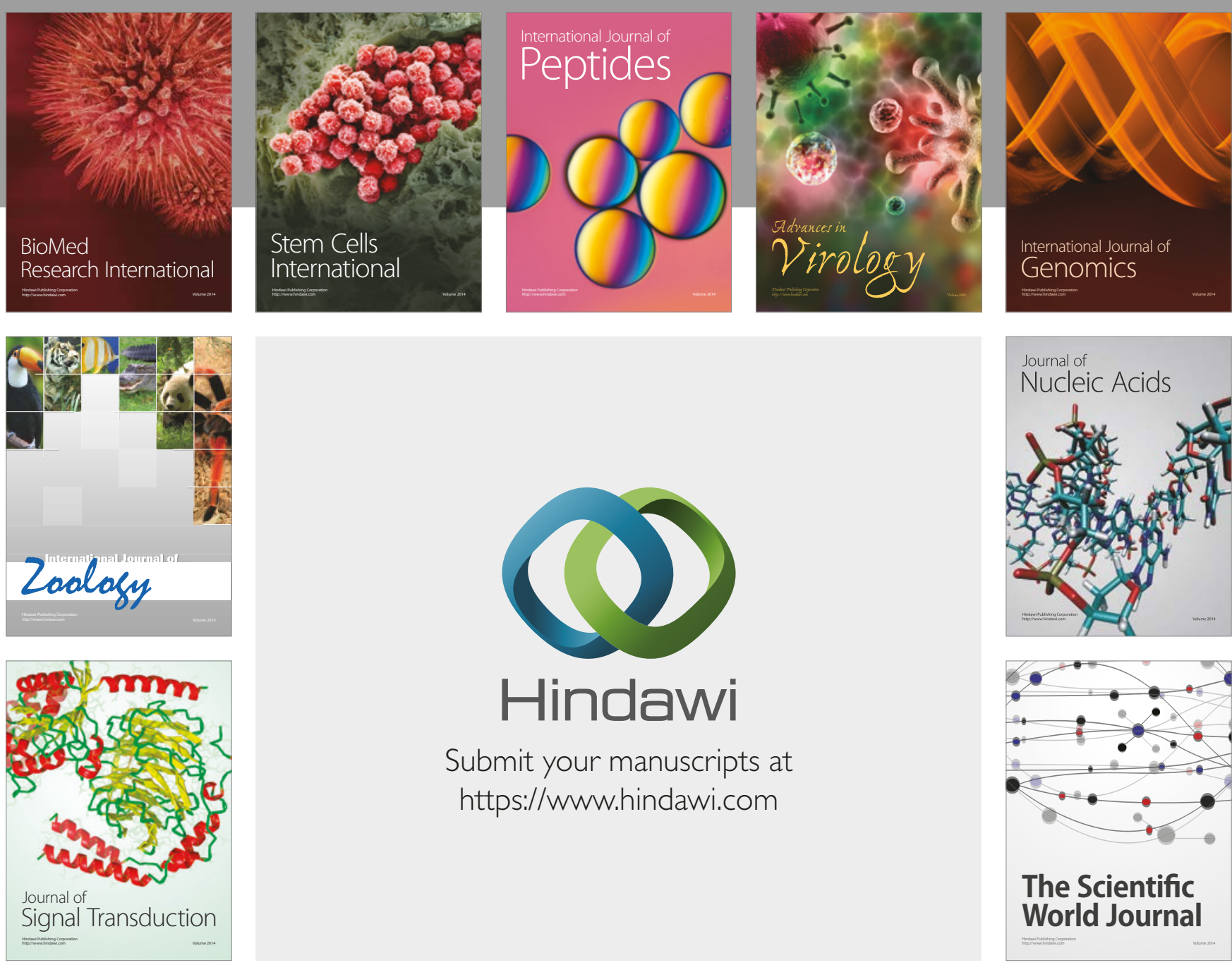

Submit your manuscripts at

https://www.hindawi.com
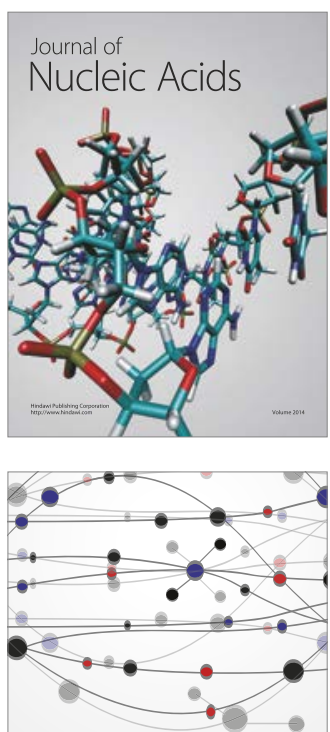

The Scientific World Journal

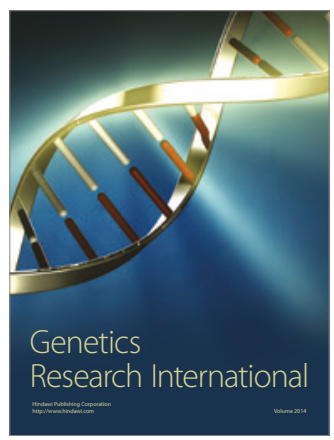

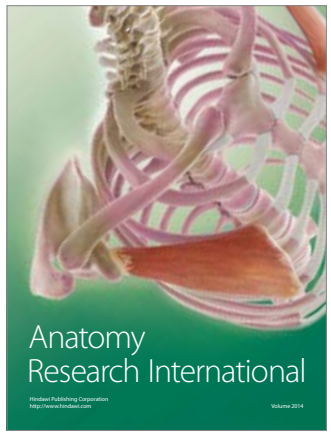

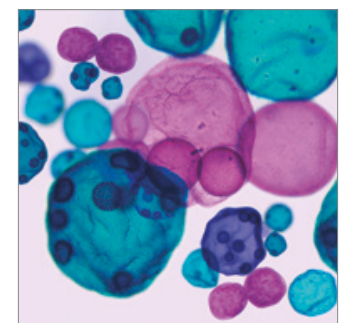

International Journal of Microbiology
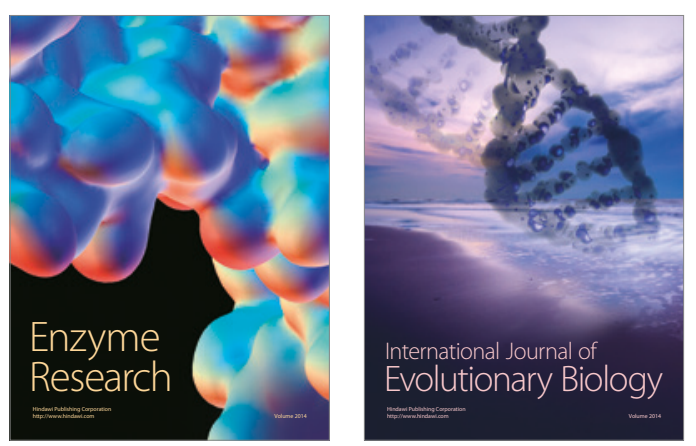
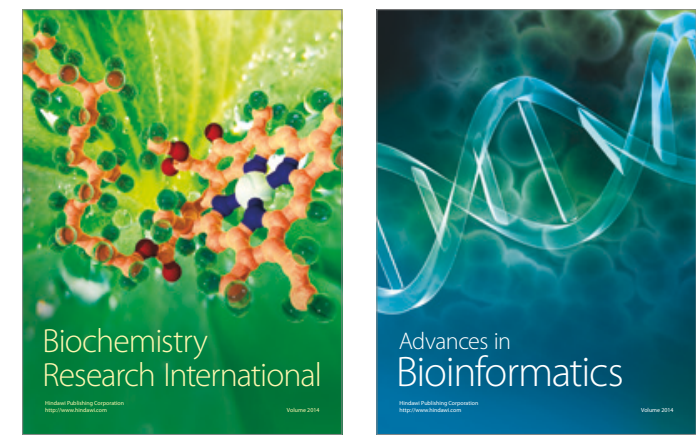

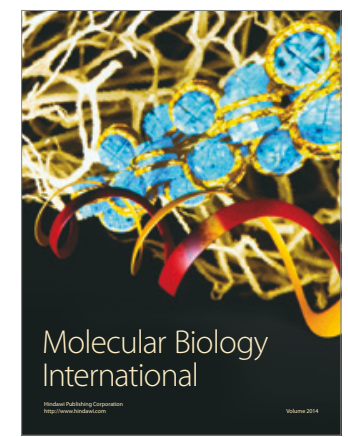

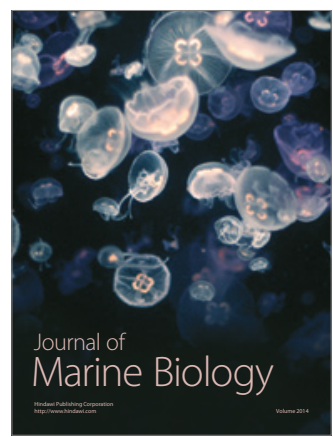

